\title{
Multidisciplinary Holistic Assessment in Diagnosis and Therapy of Autism
}

\author{
Slobodan Dunjić, J. Pejić, E. Filipović, M. Maletić, P. Kraković, V. Subotić, and S. Stanisic \\ Center for Integrative procedures and Supplements dr Dunjic, Beograd 11070, Srbija
}

\begin{abstract}
Introduction: The medical test for autism is not clearly defined. Diagnosis is based on observation of behavior and educational and psychological testing, as well as medical and laboratory analyses of various specialties. Since symptoms of autism vary, pathways leading to diagnosis are also different. It is important to know that autism is not one disorder with a unique cause. Autism is most likely the cause of combinations of genetic risk factors with risk factors from the environment. In addition to common behavioral changes, we can expect epilepsy, gastrointestinal disorders, food intolerance, sleep disorders, and sensory integration disorders. Aims: to reveal the importance of multidisciplinary and holistic approach in the diagnosis of autism, the spectrum of autistic behavior of children and medical comorbidity, with the aim of creating integrative therapy and rehabilitation by personalized holistic multidisciplinary treatment. Methods: Multidisciplinary holistic diagnostic approach to health assessment was used in 21 patients with diagnosis of autism. In the health assessment, medical, integrative procedures, laboratory analyses were used. The results were statistically processed and analyzed. Result: The protocol enrolled 21 children in the "Dunjić holistic practice". All children came with diagnosis of autism. The youngest child was 3 years old and the oldest 16 years old. Distribution by experiment was in favor of male children $95 \%$. In $65 \%$ of subjects, there were some kind of allergies, $14.2 \%$ autoimmune diseases, epilepsy in $9.5 \%$, developmental disorders at $19.0 \%$. Laboratory tests show different results and deviations from normal values, thyroid gland disorders TSH and T3, fT3, hyperprolactinemia results in 23.5\%, increased oxytocin at $14.2 \%$, osteocalcin in $28.5 \%$ of subjects. Viral infections of CMV, EBV and Herpes zoster were recorded at 9.5\%. Conclusion: Holistic protocol in diagnosis and treatment of autism plays a significant role in the multidisciplinary therapeutic treatment. Integrative approach and procedures achieve homeostasis and internal potential for success and behavioral and medical therapy. It is necessary to do research projects on some laboratory analyses that can be indicators of an early stage of autism. Early intervention has always been the key to healing in the highest percentage.
\end{abstract}

Key words: Autism, holistic, integrative medicine.

\section{Introduction}

Autism is a neurodevelopmental disorder characterized by significant damage in the area of reciprocal social interactions and communication patterns, as well as a limited, stereotypical, repetitive behavioral repertoire, interest and activity [4].

Autism is a developmental pervasive disorder with a serious form of psychic and emotional disorganization and disharmony of the personality that brings linguistic pathology, in particular linguistic retardation, as well as the general disorder of communication [5].

About $35 \%$ of children with autism are without

Corresponding author: Slobodan Dunjic, MD, Dr, research fields: integrative medicine. intellectual disabilities, $65 \%$ have intellectual disabilities at different levels [4, 5].

Research shows that about $35 \%$ of autistic subjects have normal intelligence, of which $5 \%$ are transverse.

The etiology of this disorder is basically unknown, but it is considered to have genetic basis, because it develops more frequently in single-skinned twins (90\%) than in doubles (30\%).

A number of gene loci of genes associated with autism have been recorded, but not one gene or even a gene group is not definitely associated with this disorder. Conventional medicine did a little to examine external factors that could affect the expression of autism. However, it is integrative doctors who examined the role of exposure to toxins (especially 
live), nutritional factors, infectious diseases and autoimmune diseases as factors that contribute to the onset of disease [1, 2].

Numerous doctors and researchers have dealt with a biomedical approach to autism. The foundation of this approach is that autism is a syndrome where the fetus or neonatus is exposed to a variety of early stages external factors, nutritive metabolic autoimmune, exposure toxins and electromagnetic radiation that leads to the development of the disease, but this approach also forms the basis of the treatment of disorders, solving these factors [1].

The medical test for autism is not clearly defined. Diagnosis is based on observation of behavior and educational and psychological testing, as well as medical and laboratory analyses of various specialties.

Since symptoms of autism vary, pathways leading to diagnosis are also different. It is important to know that autism is not one disorder with a unique cause. Autism is most likely the result of combinations of genetic risk factors with risk factors from the environment [1-3].

In addition to common behavioral changes, we can expect epilepsy, gastrointestinal disorders, food intolerance, sleep disorders, and sensory integration disorders [3].

\section{Aims}

It aims to show the importance of multidisciplinary and holistic approach in the diagnosis of autism, the spectrum of autistic behavior of children and medical comorbidity, with the aim of creating integrative therapy and rehabilitation by personalized holistic multidisciplinary treatment $[4,5]$.

\section{Methods}

Multidisciplinary holistic diagnostic approach to health assessment was used in 21 patients with diagnosis of autism. In the health assessment, medical, integrative procedures, laboratory analyses were used. The results were statistically processed and analyzed.

\section{Results}

The protocol enrolled 21 children in the "Dunjić holistic practice”. All children came with diagnosis of autism. The youngest child was 3 years old and the oldest 16 years old.

In $65 \%$ of subjects, there were some kind of allergies, $14.2 \%$ autoimmune diseases, epilepsy in $9.5 \%$, developmental disorders at $19.0 \%$ (Table 1 ).

Laboratory tests show different results and deviations from normal values, thyroid gland disorders TSH and T3, fT3, hyperprolactinemia results in $23.5 \%$, increased oxytocin at $14.2 \%$, osteocalcin in $28.5 \%$ of subjects. Viral infections of CMV, EBV and Herpes zoster were recorded at 9.5\% (Table 2).

Table 1 Distribution of patients with autism to comorbidity.

\begin{tabular}{lll}
\hline & $\mathrm{N}$ & $\%$ \\
\hline Allergies & 13 & 65.0 \\
Autoimmune diseases & 3 & 14.2 \\
Epilepsy & 2 & 9.0 \\
Developmental disorders & 4 & 19.0 \\
\hline
\end{tabular}

Table 2 Distribution of patients with autism according to laboratory analysis.

\begin{tabular}{lll}
\hline & $\mathrm{N}$ & $\%$ \\
\hline Thyroid gland disorders TSH and T3, Ft3, hyperprolactinemia & 5 & 23.50 \\
Increased oxytocin & 3 & 14.2 \\
Osteocalcin & 6 & 28.50 \\
Viral infections of CMV, EBV and Herpes zoster & 2 & 9.50 \\
\hline
\end{tabular}




\section{Conclusion}

Holistic protocol in diagnosis and treatment of autism plays a significant role in the multidisciplinary therapeutic treatment. Integrative approach and procedures achieve homeostasis and internal potential for success and behavioral and medical therapy.

It is necessary to do research projects on some laboratory analyses that can be indicators at an early stage of autism. Early intervention has always been the key to healing in the highest percentage.

\section{References}

[1] Zigar, D., Krstić, D., Djunic, M., Stanisic, S., Stanisic, M., Duronjic, M., Jovanovic Ignjatic, Z., and Milićević, N. 2012. "Detection of Pathological Electromagnetic Field Radiation by Using the Bi-digital O-Ring Test (BDORT).” European Journal of Integrative Medicine 4 (1): 20, 10.1016/j.eujim.2012.07.520, 2012.

[2] Stanišić, S. "Controversies in Education and Clinical Implementation of Integrative Secondary Health Science.” In Proceedings of the International Scientific Conference:
All about People: Society and Science for Integrated Care of People.11.12 mart.2016.Maribor, Alma Mater Europea ECM, -1, 1, 1, pp. 193-6, ISBN 978-961-6966-15-3.

[3] Stanišić, S., and Dunjić, M. 2013 "Diagnosis and Treatment of Food Intolerans in Autistic Children.”Presented at the 6th European Congress of Integrative Medicine.Berlin, Germany, Research in Complementary Medicine, -1, 20, 1, pp. 46-7, 1661-4119, 978-3-318-02522-4, 5, 3. - 4. Oct, 2013.

[4] Dunjić, M., Stanišić, S., Jovanović Ignjatić, Z., Stanišić, M., Jevdić, D., Zigar, D., et al. 2011 "Integrative Approach to Autism-Diagnoses and Treatment by Bi-Digital O-Ring Test.”European Journal of Integrative Medicine.4th European Congress of Integrative Medicine,Berlin, Germany, European Journal of Integrative Medicine, $-1,1,1$, p. 101, 1876-3820, 5, 2011.

[5] Dunjić, M., and Stanišić, S. 2016. “Integrative Approach in Treatment of Autismc." In Proceedings of the 4th International Scientific Conference: All about People: Society and Science for Integrated Care of People, Maribor, Alma Mater Europea ECM, -1, 1, 1, pp. 29-35, ISBN 978-961-6966-15-3. 